\title{
Quark-Lepton Symmetry and Quartification in Five Dimensions*
}

\author{
Kristian L. McDonald ${ }^{\dagger}$ \\ Research Center for High Energy Physics, University of Melbourne, \\ Victoria, 3010, Australia \\ ${ }^{\dagger}$ E-mail: k.mcdonald@physics.unimelb.edu.au
}

\begin{abstract}
We outline some features of higher dimensional models possessing a Quark-Lepton (QL) symmetry. The QL symmetric model in five dimensions is discussed, with particular emphasis on the use of split fermions. An interesting fermionic geography which utilises the QL symmetry to suppress the proton decay rate and to motivate the flavor differences in the quark and leptonic sectors is considered. We discuss the quartification model in five dimensions and contrast the features of this model with traditional four dimensional constructs.
\end{abstract}

Keywords: Extra dimensions, split fermions, quark-lepton symmetry, quartification.

\section{Introduction}

The Standard Model (SM) of particle physics displays a clear asymmetry between quarks and leptons. Quarks and leptons have different masses and charges and importantly quarks experience the strong force. Despite these differences a suggestive similarity exists between the family structure of quarks and leptons, leading one to wonder if the SM may be an approximation to a more symmetric fundamental theory.

The similar family structure of quarks and leptons is an automatic consequence of the defining symmetry of the Quark-Lepton (QL) symmetric model. ${ }^{1}$ In this framework the similarity between quarks and leptons is elevated to an exact symmetry of nature. The model permits a complete interchange symmetry between quarks and a generalized set of leptons, with the SM resulting from the breaking of an enlarged symmetry group.

On an independent front, a number of model building tools which employ extra spatial dimensions have been developed in recent years. In particular new methods of symmetry breaking have been uncovered. These methods allow one to reduce the scalar content required to achieve symmetry breaking in four dimensionsal models. It is natural to investigate these methods within pre-existing frameworks to determine

* Talk given at the Festschrift in honour of Bruce McKellar and Girish Joshi at the University of Melbourne, 14/12/2006. To appear in a special edition of IJMPA. The talk is based on the results of references ${ }^{12,17,22,29}$. 
the phenomenological distinctions between the new and traditional approaches.

In this work we outline some recent investigations in five dimensional models possessing a QL symmetry. We focus our attention on two aspects of these investigations. First we consider the use of split fermions in models with a QL symmetry. We show that the symmetry constrains the implementation of split fermions and allows one to solve some outstanding problems in four dimensional with QL models; namely how can one resolve the notion of a QL symmetry with the disimilarity between the observed masses of quark and lepton.

We also consider the quartification model, a framework which extends the notion of a QL symmetry to permit gauge unification, in five dimensions. We show that the higher dimensional quartification model allows one to remove many of the complications which arise in four dimensional models. These complications result mainly from the relatively large Higgs sector required to achieve the necessary symmetry breaking. We show that an effective Higgsless limit may be obtained in the five dimensional quartification model, thereby permitting considerable simplification.

\section{Quark-Lepton Symmetry}

How does one construct a quark-lepton symmetric model? Let us recall that the SM also displays a clear asymmetry between left and right handed fields; the left handed fields experience $S U(2)_{L}$ interactions whilst the right handed fields do not. One generation of SM fermions may be denoted as

$$
Q, L, u_{R}, d_{R}, e_{R},
$$

revealing a further left-right asymmetry; namely the absence of $\nu_{R}$. However the left-right asymmetry may be a purely low energy phenomenon and the construction of a high energy left-right symmetric theory proceeds as follows. One must first extend the SM particle content to include $\nu_{R}$ and thereby equate the number of left and right degrees of freedom. The SM gauge group must also be enlarged: ${ }^{2-5}$

$$
S U(3) \otimes S U(2) \otimes U(1) \rightarrow S U(3) \otimes[S U(2)]^{2} \otimes U(1),
$$

where the second $S U(2)$ group acts on right-chiral fermion doublets. This enables one to define a discrete symmetry interchanging all left and right handed fermions in the Lagrangian, $f_{L} \leftrightarrow f_{R}$. Furthermore the model must be constructed such that the additional symmetries introduced to enable the left-right interchange are suitably broken to reproduce the SM at low energies. In practise this means that a suitable extension of the Higgs sector is also required.

One may construct a quark-lepton symmetric model by employing the same recipe. ${ }^{1,6-11}$ First the fermion content of the SM must be extended. As quarks come in three colors one is required to introduce more leptons to equate the number of quark and lepton degrees of freedom. For each SM lepton one includes two exotic leptons,

$$
\begin{gathered}
e \rightarrow E=\left(e, e^{\prime}, e^{\prime \prime}\right), \\
\nu \rightarrow N=\left(\nu, \nu^{\prime}, \nu^{\prime \prime}\right),
\end{gathered}
$$


where the primed states are the exotics (known as liptons in the literature). The gauge group must also be extended:

$$
S U(3) \otimes S U(2) \otimes U(1) \rightarrow[S U(3)]^{2} \otimes S U(2) \otimes U(1),
$$

where the additional $S U(3)$ gauge bosons induce transitions amongst the generalized leptons in the same way that gluon exchange enables quarks to change color. Now one may define a discrete symmetry interchanging all quarks and (generalized) leptons in the Lagrangian,

$$
Q \leftrightarrow L, \quad u_{R} \leftrightarrow E_{R}, \quad d_{R} \leftrightarrow N_{R} .
$$

The gauge symmetry may be broken via the Higgs mechanism to reproduce the charge differences between quarks and leptons and give heavy masses to the liptons. Phenomenologically consistent models can be obtained by breaking the lepton color group $S U(3)_{\ell}$ completely or by leaving an unbroken subgroup $S U(2)_{\ell} \subset S U(3)_{\ell}$ (which serves to confine the liptons into exotic bound states). The QL symmetry implies mass relations of the type $m_{u}=m_{e}$ which are more difficult to rectify. In $4 \mathrm{D}$ one may remove these by extending the scalar sector and thereby increasing the number of independent Yukawa couplings.

\section{QL Symmetry in Five Dimensions}

In recent years the study of models with extra dimensions has revealed a number of new model building tools. The use of orbifolds provides a new means of reducing the gauge symmetry operative at low energies by introducing a new mass scale, namely the compactification scale $M_{c}=1 / R$. In generic 5D models the mass of exotic gauge bosons can be set by $M_{c}$ and the reduction of symmetries by orbifold construction results in collider phenomenology which differs from that obtained in models employing the usual 4D Higgs symmetry breaking.

The reduction of the QL symmetric gauge group via orbifold construction has recently been studied in 5D. ${ }^{12}$ One question that faces the model builder in extra dimensional models is whether to place fermions on a brane or in the bulk. We shall focus on the latter in what follows and demonstrate that the troublesome mass relations which arise in 4D QL models actually provide useful and interesting model building constraints in 5D models. ${ }^{13}$ We note that placing fermions in the bulk permits the unification of quark and lepton masses at $\mathrm{TeV}$ scales in more a general class of models. ${ }^{14,15}$

In five dimensions bulk fermions lack chirality. However, chiral zero mode fermions, which one may identify with SM fermions, may be obtained by employing orbifold boundary conditions on a fifth dimension forming an $S^{1} / Z_{2}$ orbifold. By coupling a bulk fermion to a bulk scalar field one may readily localise a chiral zero mode fermion at one of the orbifold fixed points. ${ }^{16}$ Denoting the bulk fermion (scalar) as $\psi(\phi)$ one has the following Lagrangian:

$$
\mathcal{L}=\bar{\psi}\left(\Gamma_{M} \partial^{M}-f \phi\right) \psi-\frac{1}{2} \partial_{M} \phi \partial^{M} \phi-V(\phi),
$$


where $\Gamma_{M}$ are the Dirac matrices, $f$ is a constant, $M=0,1 \ldots 4$ is the $5 \mathrm{D}$ Lorentz index and

$$
V(\phi)=\frac{\lambda}{4}(\phi-v)^{2},
$$

is the usual quartic potential. If $\phi$ transforms trivially under $Z_{2}$ its ground state is given by $\langle\phi\rangle=v$. However if $\phi$ is odd under $Z_{2}$ its ground state is required to vanish at the fixed points. This results in a kink vacuum profile for $\phi$ which serves to localise chiral zero mode fermions, $\psi_{0}$, at one of the orbifold fixed points. The fixed point at which $\psi_{0}$ is localised depends on the sign of $f$.

In a QL symmetric model one must specify the transformation properties of the bulk scalar under the QL symmetry. An interesting choice is to make $\phi$ odd under the QL symmetry, ${ }^{17}$ resulting in a Yukawa Lagrangian of the form

$$
\mathcal{L}=-\left\{f_{Q}\left(Q^{2}-L^{2}\right)+f_{u}\left(U^{2}-E^{2}\right)+f_{d}\left(D^{2}-N^{2}\right)\right\} \phi,
$$

where $Q^{2}=\bar{Q} Q$, etc, and the SM fermions are identified with the chiral zero modes of the bulk fermions in an obvious fashion. Note that the choice $f_{Q}, f_{u}, f_{d}>0$ automatically implies the localisation pattern shown in Figure1, It is of interest that this geography may be implemented in a less arbitrary fashion in a QL symmetric framework as this pattern is precisely that advocated recently to suppress the proton decay rate without an extremely large ultra-violet cutoff. ${ }^{18}$ Indeed the effective $4 \mathrm{D}$ proton decay inducing non-renormalizable operator has the form

$$
\mathcal{O}_{p}=\frac{K}{\Lambda^{2}} \mathcal{O}_{Q}^{3} \mathcal{O}_{L}
$$

where $\mathcal{O}_{Q}\left(\mathcal{O}_{L}\right)$ generically denotes a quark (lepton) operator and $K \sim$ $\exp \left\{-v L^{3 / 2}\right\}$ represents the wavefunction overlap between quarks and leptons in the extra dimension. $L$ denotes the length of the fundamental domain of the orbifold.

Observe that fermions related by the QL symmetry necessarily develop identical wavefunction profiles in the extra dimension and consequently the troublesome mass relations of the type $m_{e}=m_{u}$ persist in the effective $4 \mathrm{D}$ theory. With one bulk

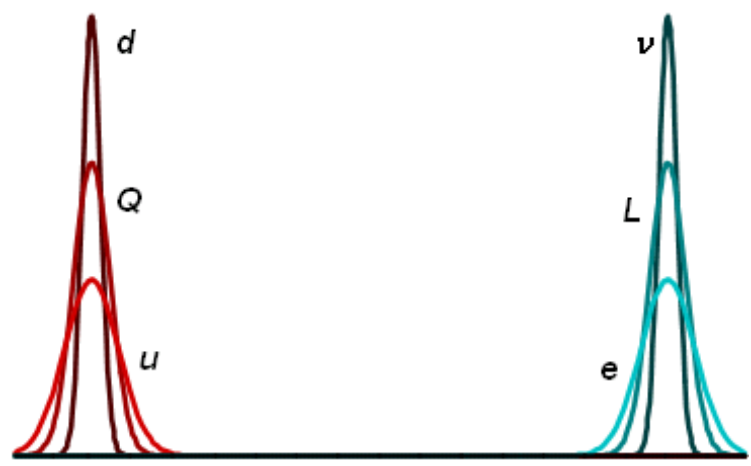

Fig. 1. The 5D wavefunctions for quarks and leptons in a QL symmetric model with one bulk scalar. 
scalar it is only possible to localise fermions at the orbifold fixed points. However two bulk scalar models enable one to localise fermions within the bulk. This works as follows. ${ }^{19,20}$ With one bulk scalar chiral zero mode fermions are found at one of the orbifold fixed points, with the precise point of localisation determined by the sign of the relevant Yukawa coupling. If a second bulk scalar is added with an opposite sign Yukawa coupling it will tend to drag the fermion towards the other end of the extra dimension, thereby localising it in the bulk. Importantly though the chiral fermion cannot be pulled very far into the bulk before it is dragged all the way to the other end of the extra dimension.

Let us add a second bulk scalar which is even under the QL symmetry, giving rise to the Lagrangian

$$
\begin{gathered}
\mathcal{L}_{2}=-\left\{h_{Q}\left(Q^{2}+L^{2}\right)+h_{u}\left(U^{2}+E^{2}\right)\right. \\
\left.+h_{d}\left(D^{2}+N^{2}\right)\right\} \phi^{\prime} .
\end{gathered}
$$

Let us again require $h_{Q}, h_{u}, h_{d}>0$ so that all quark Yukawa couplings are positive. Both $\phi$ and $\phi^{\prime}$ will attempt to localize quarks at the same point in the extra dimension and they will remain at their original point of localisation. This type of quark geography is precisely that recently advocated ${ }^{23}$ to allow one to construct quark flavor without introducing large flavor changing neutral currents. However $\phi$ and $\phi^{\prime}$ attempt to localise leptons at different fixed points and the resultant point of localisation for a given lepton depends on which scalar dominates (these statements will be made numerically precise in a forthcoming publication $\left.{ }^{21}\right)$. An arrangement typical of this setup is shown in Figure 2 .

This arrangement has a number of interesting features. Firstly note that shifting leptons into the bulk significantly alters the degree of wavefunction overlap in the quark and leptonic sectors. When one obtains the effective 4D fermion masses the different degree of overlap in the quark and lepton sectors will remove the undesirable QL mass relations. Furthermore the overlap between left and right chiral fermion wavefunctions is expected to be greater in the quark sector than in the

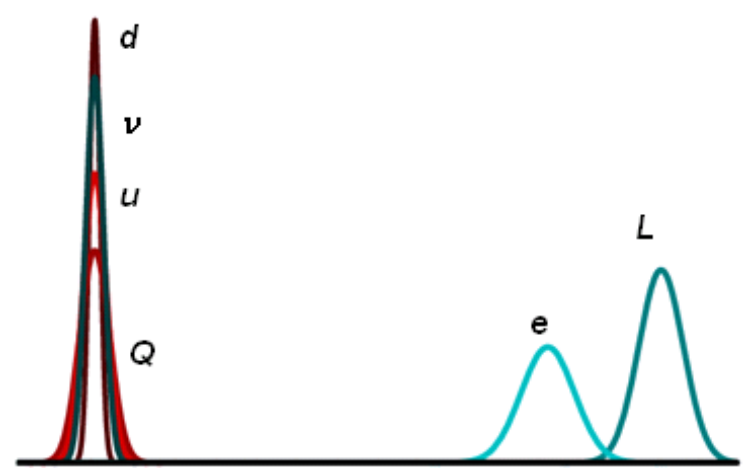

Fig. 2. The 5D wavefunctions for quarks and leptons in a QL symmetric model with two bulk scalars. 
lepton sector, leading to the generic expectation that quarks will be heavier than the lepton to which they're related by the QL symmetry. Observe that dragging the right chiral neutrinos $\nu_{R}$ all the way to the 'quark end' of the extra dimension significantly suppresses the neutrino Dirac masses below the electroweak scale. Dragging $\nu_{R}$ to the quark end of the extra dimension does not introduce rapid proton decay, provided that $\nu_{R}$ has a large enough Majorana mass to kinematically preclude decays of the type $p \rightarrow \pi \nu_{R}$. Such a mass is expected to arise at the non-renormalizable level even if it is not induced by tree-level couplings in the theory. ${ }^{22}$

Thus the non-desirable Yukawa relations implied by 4D QL symmetric models turn out to be of interest in the 5D construct. They enable one to understand proton longevity within the split fermion framework in a less arbitrary fashion and instead of inducing unwanted mass relations suggest an underlying motivation for the flavour differences experimentally observed in the quark and lepton sectors.

\section{Quartification in Five Dimensions}

Adding a leptonic color group to the SM clearly renders the traditional approaches to gauge unification inapplicable. Recall that the simplest grand unified theory, namely $S U(5)$, does not contain the left-right symmetric model. However there are larger unifying groups which do admit the left-right symmetry, for example $S O(10)$ and the trinification gauge group $[S U(3)]^{3} \times Z_{3}$. Similarly it is possible to construct a unified gauge theory admitting the QL symmetry by considering larger unification groups. It is natural to extend the notion of trinification to include a leptonic color factor, leading one to the so called quartification model. This possesses the gauge group $G_{Q}=[S U(3)]^{4} \times Z_{4}$, where the additional $S U(3)$ factor corresponds to lepton color and the $Z_{4}$ symmetry cyclicly permutes the group factors to ensure a single coupling constant. ${ }^{24-29}$

It was been demonstrated that unification may be achieved within the quartification framework in $4 \mathrm{D}$ by enforcing additional symmetries upon the quartification model. ${ }^{25}$ Subsequent work has shown that unification need not require additional symmetries, but does require multiple symmetry breaking scales between the unification scale and the electroweak scale. ${ }^{27}$ It was also shown ${ }^{27}$ that unification may be achieved via multiple symmetry breaking routes both with and without the remnant leptonic color symmetry $S U(2)_{\ell}$.

The necessary symmetry breaking is accomplished with eight Higgs multiplets, giving rise to a complicated Higgs potential with a large number of free parameters. The demand of multiple symmetry breaking scales also requires hierarchies of vacuum expectation values (VEV's) to exist within individual scalar multiplets, giving rise to a generalized version of the doublet-triplet splitting problem familiar from $S U(5)$ unified theories. A large number of electroweak doublets also appear in the $4 \mathrm{D}$ constructs. Thus many of the less satisfactory features of $4 \mathrm{D}$ quartification models revolve around the Higgs sector required for symmetry breaking.

Recently the quartification model has been studied in 5D, where intrinsically 
higher dimensional symmetry breaking methods exist. ${ }^{29}$ By taking the fifth dimension as an $S^{1} / Z_{2} \times Z_{2}^{\prime}$ orbifold one may employ orbifold boundary conditions (OBC's) on the bulk gauge sector to reduce the gauge symmetry operative at the zero mode level from $G_{Q}$ to

$$
S U(3)_{c} \otimes S U(2)_{L} \otimes S U(2)_{\ell} \otimes S U(2)_{R} \otimes U(1)^{3} .
$$

However the use of OBC's does not reduce the rank of the gauge group so that further symmetry breaking is required. Rank reducing symmetry breaking can be achieved in higher dimensional theories by employing a boundary scalar sector to alter the boundary conditions on the compactified space for gauge fields. ${ }^{30}$ Denoting a boundary scalar as $\chi$ and defining $V \propto\langle\chi\rangle$ one can show that $V$ induces a shift in the Kaluza-Klein mass spectrum of gauge fields which couple to $\chi$. If such a gauge field initially possessed a massless mode its tower receives a shift of the form

$$
M_{n} \approx M_{c}(2 n+1)\left(1+\frac{M_{c}}{\pi V}+\ldots\right), \quad n=0,1,2, \ldots
$$

giving a tower with the lowest-lying states $M_{c}, 3 M_{c}, 5 M_{c}, \ldots$. This represents an offset of $M_{c}$ relative to the $V=0$ tower, with the field no longer retaining a massless zero mode. The association of $V$ with the VEVs of the boundary scalar sector implies that the limit $V \rightarrow \infty$ is attained when $\langle\chi\rangle \rightarrow \infty$. However, when the VEVs of the Higgs fields are taken to infinity, the shift in the KK masses of the gauge fields is finite, giving the exotic gauge fields masses dependent only upon the compactification scale $M_{c}$. Consequently, these fields remain as ingredients in the effective theory while the boundary Higgs sector decouples entirely, and we can view our reduced symmetry theory in an effective Higgsless limit. Interestingly, in this limit also, the high-energy behaviour of the massive gauge boson scattering remains unspoilt as shown in. ${ }^{30}$

It was shown in $^{29}$ that a unique set of OBC's was required to ensure that quark masses could be generated and to prevent liptons from appearing at the electroweak scale. The inclusion of a boundary Higgs sector allows one to reduce the quartification gauge symmetry down to the SM gauge group $G_{S M}$ or to $G_{S M} \otimes S U(2)_{\ell}$ at the zero mode level. In both cases fifth dimensional components of the $S U(3)_{L}$ gauge fields with the quantum numbers of the SM Higgs doublet retained a massless mode, enabling one to use Wilson loops to reproduce the SM flavour structure.

A surprising result however was that unification could only be achieved when the remnant lepton color symmetry $S U(2)_{\ell}$ remained unbroken. Thus one arrives at a unique minimal quartification model which unifies in $5 \mathrm{D}$, a result to be contrasted with the $4 \mathrm{D}$ case where a large number of symmetry breaking routes which permit unification have been uncovered. Unfortunately the unifying case requires the compactification scale to be greater than $10^{10} \mathrm{GeV}$ so that only a SM like Higgs field is expected to appear at the LHC. 


\section{Conclusion}

Quark-lepton symmetric models in some sense unify the fermionic content of the SM and thereby motivate the similar family structures observed in the quark and lepton sectors. Recent investigations involving QL symmetries in 5D have uncovered a number of interesting results. In particular the QL symmetry provides useful Yukawa relationships in split fermion models and the quartification model is found to be more constrained in 5D. A number of avenues for further investigation remain, including a detailed analysis of the fermionic geography of Figure $2^{21}$ and the construction of a complete three generational model with combined QL and left-right symmetry in $5 \mathrm{D} .{ }^{31}$

\section{References}

1. R. Foot and H. Lew, Phys. Rev. D 41 (1990) 3502.

2. J. C. Pati and A. Salam, Phys. Rev. D 10, 275 (1974).

3. R. N. Mohapatra and J. C. Pati, Phys. Rev. D 11, 566 (1975).

4. R. N. Mohapatra and J. C. Pati, Phys. Rev. D 11, 2558 (1975).

5. G. Senjanovic and R. N. Mohapatra, Phys. Rev. D 12, 1502 (1975).

6. R. Foot and H. Lew, Phys. Rev. D 42 (1990) 945.

7. R. Foot and H. Lew, Mod. Phys. Lett. A 5, 1345 (1990).

8. R. Foot, H. Lew and R. R. Volkas, Phys. Rev. D 44, 1531 (1991).

9. Y. Levin and R. R. Volkas, Phys. Rev. D 48, 5342 (1993) arXiv:hep-ph/9308256.

10. D. S. Shaw and R. R. Volkas, Phys. Rev. D 51, 6490 (1995) arXiv:hep-ph/9410350].

11. R. Foot and R. R. Volkas, Phys. Lett. B 358, 318 (1995) arXiv:hep-ph/9505331.

12. K. L. McDonald and B. H. J. McKellar, Phys. Rev. D 74, 056005 (2006) arXiv:hep-ph/0609110.

13. A. Coulthurst, A. Demaria, K. L. McDonald and B. H. J. McKellar, arXiv:hep-ph/0611269

14. P. Q. Hung, Nucl. Phys. B 720 (2005) 89 arXiv:hep-ph/0412262.

15. M. Adibzadeh and P. Q. Hung, arXiv:0705.1154 [hep-ph].

16. H. Georgi, A. K. Grant and G. Hailu, Phys. Rev. D 63, 064027 (2001) arXiv:hep-ph/0007350.

17. A. Coulthurst, K. L. McDonald and B. H. J. McKellar, Phys. Rev. D 74, 127701 (2006) arXiv:hep-ph/0610345.

18. N. Arkani-Hamed and M. Schmaltz, Phys. Rev. D 61, 033005 (2000) arXiv:hep-ph/9903417.

19. Y. Grossman and G. Perez, Phys. Rev. D 67, 015011 (2003) arXiv:hep-ph/0210053.

20. Y. Grossman and G. Perez, Pramana 62, 733 (2004) arXiv:hep-ph/0303243].

21. A. Coulthurst and K. L. McDonald, in preparation.

22. A. Coulthurst, K. L. McDonald and B. H. J. McKellar, Phys. Rev. D 75, 045018 (2007) arXiv:hep-ph/0611164.

23. B. Lillie, JHEP 0312, 030 (2003) arXiv:hep-ph/0308091.

24. G. C. Joshi and R. R. Volkas, Phys. Rev. D 45, 1711 (1992).

25. K. S. Babu, E. Ma and S. Willenbrock, Phys. Rev. D 69, 051301 (2004) arXiv:hep-ph/0307380.

26. S. L. Chen and E. Ma, Mod. Phys. Lett. A 19, 1267 (2004) arXiv:hep-ph/0403105.

27. A. Demaria, C. I. Low and R. R. Volkas, Phys. Rev. D 72, 075007 (2005) arXiv:hep-ph/0508160. 
28. A. Demaria, C. I. Low and R. R. Volkas, Phys. Rev. D 74, 033005 (2006) arXiv:hep-ph/0603152.

29. A. Demaria and K. L. McDonald, Phys. Rev. D 75, 056006 (2007) arXiv:hep-ph/0610346.

30. C. Csaki, C. Grojean, H. Murayama, L. Pilo and J. Terning, Phys. Rev. D 69, 055006 (2004) arXiv:hep-ph/0305237.

31. A. Coulthurst, J. Doukas and K. L. McDonald, arXiv:hep-ph/0702285. 